\title{
Carinal resection
}

\section{Lorenzo Spaggiaria, ${ }^{a}$, Francesco Petrella ${ }^{a}$, and Domenico Galetta ${ }^{a}$}

\author{
aDivision of Thoracic Surgery, European Institute of Oncology, Milan, Italy \\ bUniversity School of Milan, Milan, Italy \\ * Corresponding author. Division of Thoracic Surgery, European Institute of Oncology, Via Ripamonti 435, Milan, Italy. Tel: +39-02-57489665; \\ fax: +39-02-54789698; e-mail: lorenzo.spaggiari@ieo.it (L. Spaggiari).
}

Received 26 April 2011; received in revised form 23 August 2011; accepted 4 September 2011

\begin{abstract}
Summary
Carinal resection is defined as the resection of tracheo-bronchial bifurcation, with or without lung parenchyma resection. It represents one of the most challenging areas of airway resection and reconstruction, basically due to the variability in the location and extent of the lesions. Main indications for this procedure are primary tumours of the carina or the distal trachea or, more frequently, bronchogenic carcinoma with carinal involvement. Very different approaches and reconstruction techniques have been experimentally and clinically described in the last 50 years, with some corner stone procedures in the history of modern thoracic surgery. Despite many technical and oncological difficulties encountered in this field, encouraging results have been reported in recent series, in particular an excellent 5-year survival rate of $50 \%$ in pN0 patients suffering form carinal infiltration form lung cancer. Several aspects of the multimodality approach to neoplastic carinal involvement still remain debatable like radio-chemotherapeutic approach instead of the extremely rare left carinal pneumonectomy as well as the role of induction treatments before embarking in such demanding procedures, according to the pathological nodal status.
\end{abstract}

Keywords: Airways • Carinal resection $•$ Trachea $\cdot$ Lung cancer

\section{INTRODUCTION}

Carinal resection represents one of the most challenging areas of airway resection and reconstruction; considering the variability in resection and reconstruction according to the extent of the lesion, we may say that no single ideal technique exists for reconstruction [1].

We define 'carinal resection' as the resection of tracheo-bronchial bifurcation with or without lung parenchyma resection.

Main indications are primary tumours of the carina or the distal trachea or, more frequently, bronchogenic carcinoma involving the carina or the distal trachea.

Right tracheal sleeve pneumonectomy represents the most frequent procedure, whereas left tracheal sleeve pneumonectomy or right upper lobectomy with carinal resection is infrequent.

Isolated carinal resection with tracheo-bronchial reconstruction without lung resection is very rare and its indication, from the oncological point of view, is limited to localized primary tumours of the carina or the distal trachea.

\section{History}

Experimental resection of tracheal carina started during the late 40 s and continued for almost 20 years [1]; Grindlay et al. [2] first resected right lung and carina in dogs in 1949, with end-to-end anastomosis of the trachea to the left main bronchus. Juvenelle and Citret [3] performed experimentally lateral implantation of the bronchus (right or left) into the trachea after a short tracheal segment resection. Subsequently, several experimental studies showed the feasibility of bronchial implantation into the trachea, bronchial transposition to the lateral tracheal wall as well as some carinal reconstruction techniques including reapproximation of both main bronchi to the carina and tracheo-bronchial anastomoses after carinal pneumonectomy $[4,5]$.

The first clinical carinal resection was performed by Abbott [6], repairing a large oval defects created by a right pneumonectomy and right carinal lateral excision for bronchogenic carcinoma in 1950 by transverse closure.

In 1951, Mathey [7] locally resected a cylindroma of the carina involving posterior walls of both proximal main bronchi; reconstruction was performed by a longitudinal suture of the medial bronchi margins and a transverse suture of the remaining defect.

In 1954, Craaford et al. [8] performed an anastomosis between the bronchus intermedius and the trachea, at the origin of the right main bronchus, after upper lobectomy and bronchial excision.

In 1955, Bjork [9] exposed the carina from the left hemithorax, mobilizing the aorta after division of four pairs of intercostal arteries, to resect the left main bronchus and to anastomose its lobar bifurcation to the prior origin of the bronchus at the trachea.

In 1957, Barclay et al. [10] resected distal trachea and carina to remove a recurrent adenoid cystic carcinoma; the trachea was end-to-end anastomosed to the right main bronchus whereas the left main bronchus was anastomosed end-to-side to the bronchus intermedius.

In 1963, Grillo et al. [11] described resection of the distal trachea and carina to remove adenoid cystic carcinoma; the trachea was end-to-end anastomosed to the right main bronchus, whereas the left main bronchus was anastomosed end-to-side to the trachea. 
In 1961, Nissen [12] removed a malignant adenoma, while Woods et al. [13] excised a cylindroma of the carina by using extracorporeal circulation; reconstruction was done by suturing and using a skin patch supported by a wire mesh.

In 1967, Eschapasse et al. [14] reported a short series of three patients receiving circumferential resection of the entire carina followed by complete reconstruction; in two cases, the anastomoses were end-to-end between right main bronchus and trachea, followed by an end-to-side anastomosis between left main bronchus and bronchus intermedius. In the other case, end-to-end anastomosis was done between distal trachea and left main bronchus, followed by an end-to-side anastomosis between intermediate bronchus and the left main bronchus.

In 1982, on the basis of his experience, Grillo [15] presented a comprehensive schema for carinal reconstruction: for short resections, carinal restoration was by side-to-side main bronchial anastomosis, which was then joined end-to-end to the trachea; for longer lesions, the trachea was placed end-to-end to the left main bronchus, if the gap was $<4 \mathrm{~cm}$, and the right main bronchus endto-side to the trachea; for still more extensive tracheo-carinal removal, the 'Barclay' anastomosis of the right main bronchus to the trachea and left main bronchus end-to-side to the intermediate bronchus was used.

In more recent years, thanks to bioengineering and stem cells therapy, there have been interesting developments in the field of tracheal transplantation and bioengineered airway tissue employment: in 2004, Klepetko et al. [16] reported a case of heterotopic tracheal transplantation with omentum wrapping in the abdominal position; in the same year, Macchiarini et al. [17] reported the first human transplantation of a bioengineered airway tissue to treat a complete dehiscence of the ventro-lateral aspect of the tracheo-bronchial anastomosis after a right completion carinal pneumonectomy.

In 2008, Macchiarini et al. [18] reported the complete resection of the left main bronchus for non-neoplastic disease and its successful replacement with a bioengineered human trachea, by left posterolateral thoracotomy, to avoid left carinal pneumonectomy.

\section{Approaches to the carina}

The surgical approach to the carina may be performed by a lateral or a postero-lateral thoracotomy or by a median sternotomy, according to the surgeon's experience, the tumour location and the type of surgical procedure.

\section{Right thoracotomy}

It probably represents one of the best ways to approach the carina and therefore it is one of the favourite accesses by most surgeons; it is particularly indicated for tumours of the carina and for more extensive tumours of the lower trachea [15].

\section{Left thoracotomy}

It may be used for treating a tumour involving the carina as well as the left main bronchus to such an extent that the left lung cannot be salvaged; tracheal excision must be very limited when this approach is used. Left thoracotomy with subaortic dissection was principally employed for lesions involving the left main bronchus and the carina, but little of the tracheal length [15]; left thoracotomy with retro aortic dissection was also explored but failed to gain acceptance $[9,19]$; in fact, swinging the arch of the aorta forward from the left after dividing four upper intercostal arteries, as described by Bjork [9], does not provide good access for tracheal procedures [20].

\section{Median sternotomy}

Median sternotomy was first described by Goeltz [21] in 1907 for foreign body removal, was then reported in 1960 by Padhi and Lynn [22] for bronchopleural fistula and, in 1961, by Abruzzini for treatment of postpneumonectomy tubercolous fistulae [23]; it was then re-introduced with anterior and posterior pericardial opening by Perelman [24]: in this trans-mediastinal approach to the entire trachea, the sternum is fully divided; the anterior pericardium is opened vertically between the superior vena cava and the aorta; the posterior pericardium is similarly opened; retraction of the superior vena cava and the aorta exposes a quadrilateral space in which the lower trachea and the carina are seen; the right pulmonary artery lies just below the carina.

\section{Clamshell incision}

Grillo [15] employed bilateral thoracotomy for free access to the carina and to both thoraces for treatment of complex lesions, especially those involving the left main bronchus, carina and a long extent of the lower trachea.

\section{Left hemiclamshell incision}

Maeda et al. [20] added left anterior thoracotomy to median sternotomy to improve access in the case of left carinal pneumonectomy.

\section{Anatomy}

The carina is the apex of the bifurcation point of the trachea; it is located at the lower border of the T4 vertebra in the supine position and T5 or T6 vertebrae in the erect position. At the carinal level, the left main bronchus passes beneath the aortic arch and the right main bronchus beneath the azygos vein. The superior vena cava lies just anterior and to the right of the trachea; the pulmonary artery lies inferiorly in front of the carina; thus, in anterior approach to the carina, a deep quadrilateral space is developed trans-pericardially in front of the carina, bordered by the superior vena cava on the right, the aortic arch on the left, the pulmonary artery inferiorly and the brachiocephalic vessels superiorly [1].

The attachments of connective tissue to the trachea are loose enough so that vertical movement is easily possible to a considerable degree both functionally and surgically; the trachea is fixed by the sling of the aortic arch over the left main bronchus where relatively little sliding motion occurs [1].

The bronchial arteries provide blood supply to the carina and the lowermost trachea; the bronchial arterial circulation may be divided into anterior branches, arising from the subclavian, internal mammary, or coronary arteries, and posterior branches, arising from the thoracic aorta or intercostal arteries. Anterior branches are uncommon and assume clinical significance only in instances in which the posterior branches are interrupted, such as following en bloc heart-lung transplantation. In these instances, anterior branches, particularly from the coronary circulation, provide significant systemic collateral blood flow to the airway for anastomotic healing and maintenance of structural integrity of the tracheo-bronchial tree [25].

The branches of the posterior circulation are divided into leftand right-sided arteries. A left bronchial artery arises directly from the descending thoracic aorta in more than $90 \%$ of cases. A right bronchial artery arises in relationship to a right intercostal artery as a right inter-costo-bronchial artery (RICBA) in $95 \%$ of cases. Additional right- and left-sided bronchial arteries may arise 
directly from the aorta or from ipsilateral intercostal arteries. The most common patterns of posterior bronchial circulation consist of one right and two left bronchial arteries (in $~ 25 \%$ of patients) or two right and two left bronchial arteries (in $\sim 20 \%$ of patients). The RICBA arises from the descending thoracic aorta, crossing posterior to the oesophagus and then passing between the azygous vein and the oesophagus to the right main bronchus. It serves as a common trunk for the first or second right intercostal artery and the right bronchial artery. The RICBA supplies the right main bronchus and carina, and may supply the left bronchial tree as well, through subcarinal collaterals [1].

Although a large part of the length of the trachea can usually be circumferentially dissected without necrosis if the trachea remains intact-and with it the vertical longitudinal vessels-circumferential dissection of an excessively long segment of the trachea above or below a point of tracheal division can lead to devascularization; necrosis may follow [1].

Primary tracheal lymph nodes are pretracheal, paratracheal and subcarinal [26]; the right lower paratracheal lymph nodes drain into thoracic duct tributaries which travel along the course of the azygous vein. Left superior bronchial nodes below the trachea drain directly to the mediastinal thoracic duct or to the arch of the duct via the left recurrent chain. An alternative pathway is to the aortic arch node and up along the arch. Tracheal bifurcation nodes drain through accessory ducts on either side of the oesophagus to the mediastinal thoracic duct [27].

Mediastinal lymph node dissection for tracheal tumours should be limited to lymph nodes immediately adjacent to the segment to be resected, to avoid contributing to devascularization [1].

\section{SURGICAL PROCEDURE}

\section{Carinal resection without pulmonary resection}

\section{Neocarina (approximation of the median walls of the main bronchi)}

This reconstruction is indicated in case of very small tumours, implanted on the carina only. The medial walls of the main bronchi are approximated together to fashion a new carina that is then anastomosed to the trachea [28].

The main problem with this reconstruction is that the neocarina has a very limited mobility: in fact, once the right and left main bronchi are sutured together, the left bronchus is held by the halter of the aortic arch; for this reason, most of the length for re-approximation must come from devolution of the trachea to the newly formed bifurcation [1].

Barclay (end-to-end right main bronchus with trachea; end-to-side left main bronchus with intermedius)

This reconstruction is indicated in case of more extensive tumours, requiring a larger portion of the distal trachea to be resected.

The procedure is realized into three different stages: (i) mediastinal approach to the carina throughout a median sternotomy is performed; (ii) an end-to-end anastomosis between the trachea and the right main bronchus is performed; (iii) an ovoid opening is made in the medial wall of the bronchus intermedius and an end-to-side anastomosis of the left main bronchus, across the mediastinum, into the bronchus intermedius is performed.

This reconstruction can safely be done only if the right main bronchus remains sufficiently long; moreover, it may be very difficult to perform the end-to-side anastomosis using cross-filed anaesthesia and often requires hypoventilation of the right lung $[1,28]$.

\section{Grillo (end-to-end right main into trachea; left main into lateral trachea)}

This reconstruction has the same indications as for the Barclay's technique. An end-to-end anastomosis is performed between the right main bronchus and the trachea; an end-to-side anastomosis is then performed between the left main bronchus and the lateral wall of the trachea.

This procedure is very rarely indicated and is technically very demanding [28]; Grillo himself reports that there is no special reason to recommend this scheme [1].

\section{Eschapasse (reverse Barclay: end-to-end left main with the trachea; end-to-side right main with the trachea [or left main])}

This reconstruction is indicated when a tumour involves more than a minimal amount of the trachea. The first part of the procedure is the median sternotomy to access the mediastinum. The anterior pericardial surface is opened; the ascending aorta is encircled and pulled to the left; the superior vena cava is encircled and pulled to the right. Right pulmonary artery is encircled medially to the superior vena cava and bilateral paratracheal lymph node dissection is completed (Video 1). Right main bronchus is encircled as well as the distal trachea; in this way, the carina is then exposed (Video 2). The distal trachea is transected at the level where the anastomosis with the left main bronchus is supposed to be done; a traction stitch in the distal part of the remaining trachea is passed to avoid cranial migration. An end-to-end anastomosis between the distal trachea and the left main bronchus is first performed by a 3/0 polypropylene running suture, while the right main bronchus is ventilated by cross-field intubation (Video 3). In some cases (haemodynamic instability or oxygenation problems), cross-field intubation is avoided by the use of cardiopulmonary bypass. Then, after advancing the endotracheal tube into the left main bronchus, an opening on the right aspect of the tracheal wall is performed, $1 \mathrm{~cm}$ above the previous anastomosis. An end-to-side anastomosis between the trachea and the right main bronchus is performed as described previously. Then, the water submersion test is performed to check for air-leaks and bilateral pulmonary re-inflation is completed. Finally, medial sternotomy is routinely closed (Video 4).

On rare occasions, the second anastomosis may be performed between the right main bronchus and the medial wall of the left main one, depending on the relative intraoperative tension $[1,28]$.

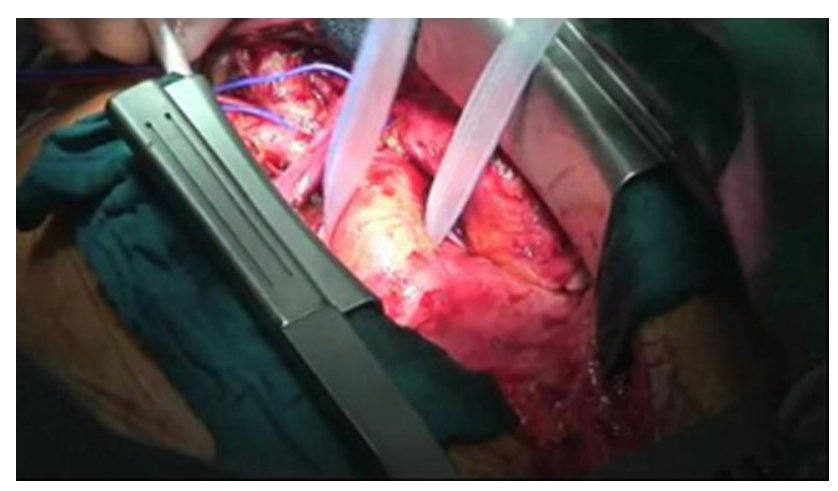

Video 1: Median sternotomy is performed to access the mediastinum and, after the pericardial opening, the mediastinal vessels are isolated and bilateral paratracheal lymph node dissection is completed. 


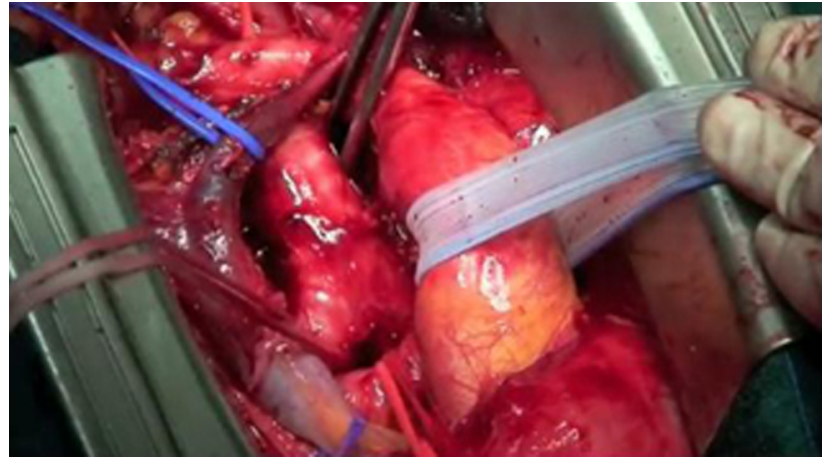

Video 2: The right main bronchus and the distal trachea are isolated and the carina is then exposed.

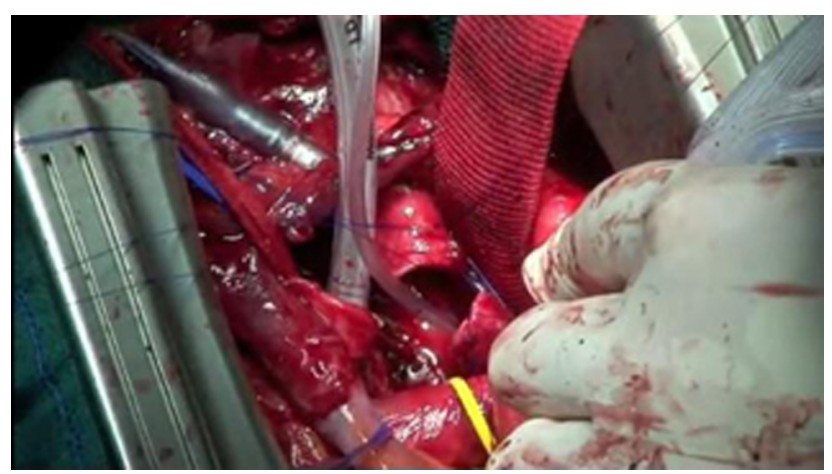

Video 3: The distal trachea is transected and a stitch is passed in the distal part of the remaining trachea to avoid cranial migration. Then, an end-to-end anastomosis between the distal trachea and the left main bronchus is performed by a $3 / 0$ polypropylene running suture and the right main bronchus is ventilated by cross-field intubation.

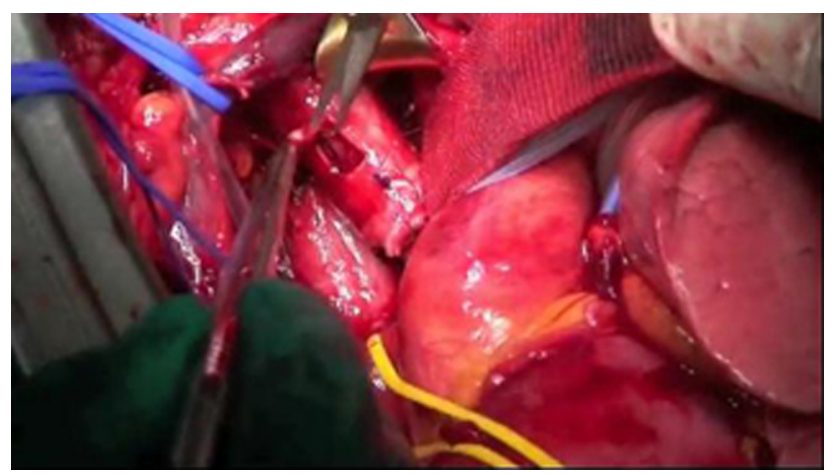

Video 4: An opening on the right aspect of tracheal wall is performed above the previous anastomosis and an end-to-side anastomosis between the trachea and the right main bronchus is performed. The suture is checked for air-leaks by water submersion test and bilateral pulmonary re-inflation is completed. Sternotomy is routinely closed.

\section{Carinal resection with pulmonary resection}

\section{Right carinal pneumonectomy}

The most common indication for this procedure is bronchogenic carcinoma involving the origin of the main bronchus or the carina.

Mediastinoscopy should be performed prior to embarking upon such a resection to be certain that the patient is potentially

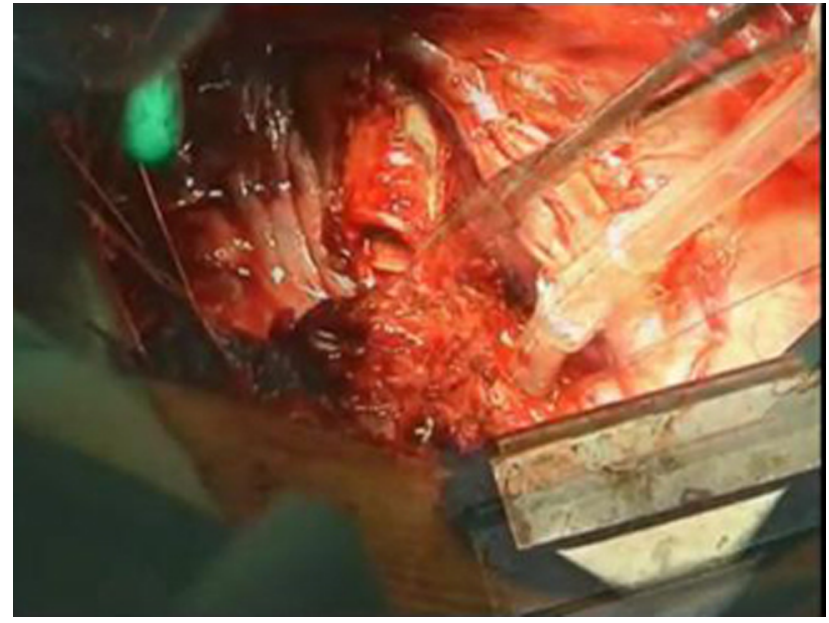

Video 5: Throughout a lateral thoracotomy at the fourth intercostal space, the pericardium is opened and the trachea circumferentially dissected. If the superior vena cava is infiltrated by the tumour, a vascular resection and reconstruction is first performed. The left main bronchus is usually divided first followed by the transection of the distal trachea, while left lung ventilation is assured by cross-field intubation of the left main bronchus.

curable [1]. The procedure is considered technically feasible when no more than $4 \mathrm{~cm}$ of the trachea will have to be resected.

This procedure is usually performed by a lateral thoracotomy in the fourth intercostal space. The pericardium is opened and the trachea dissected. If the superior vena cava is infiltrated by the tumour, a vascular resection and reconstruction-usually by means of heterologous (bovine) pericardial conduit-is first performed. The trachea is circumferentially dissected at the level of the expected division. Dissection must be done carefully to avoid injury of the left recurrent laryngeal nerve where it lies beneath and on the aortic arch [1]. The left main bronchus is usually divided first followed by the transection of the distal trachea while left lung ventilation is assured by cross-field intubation of the left main bronchus (Video 5) [1]. End-to-end anastomosis between the distal trachea and the left main bronchus is performed; crossfield intubation is maintained until the anastomosis is almost completed. Reinforcing further single stitches are passed to optimize a tension-free anastomosis. A pericardial fat patch is prepared and then interposed between vascular and bronchial anastomoses. The pericardial defect is closed by a bovine pericardial prosthesis (Video 6).

\section{Left carinal pneumonectomy}

This procedure can be performed through left thoracotomy when a limited amount of trachea is involved, more specifically when no more than $1 \mathrm{~cm}$ of trachea and of right main bronchus will have to be resected above and below the margins of the left main bronchus $[15,20,29]$.

Dissection is carried out beneath the aortic arch identifying and preserving the left laryngeal recurrent nerve; the ligamentum arteriosum is divided to improve exposure; the aortic arch is dissected circumferentially and retracted by tapes placing; the lowermost end of the trachea is dissected and then isolated after cervical flexion to devolve the trachea; the pretracheal plane is bluntly dissected into the neck; after identifying the carina, right main bronchus is dissected an then isolated; finally, being sure that resection and reconstruction are feasible, pulmonary vessels are divided [1]. 


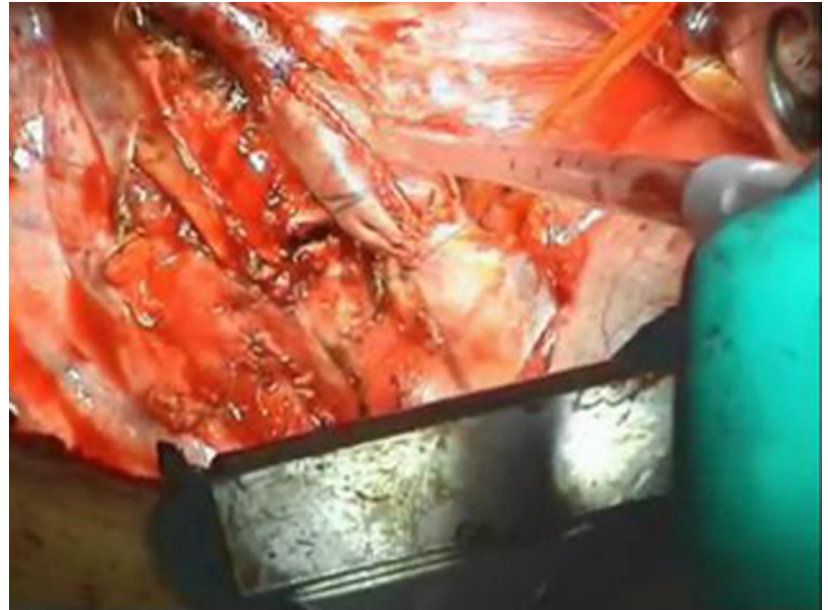

Video 6: The anastomosis between the trachea and the left main bronchus (endto-end anastomosis) is performed and cross-field intubation in the left main bronchus is maintained until the anastomosis is almost completed. Some single reinforcing stitches are used to reduce the tension at the level of anastomosis. The two anastomoses are protected by the interposition of a pericardial fat patch and the pericardial defect is closed by a heterologous prosthesis. Surgical specimen is shown, disclosing the resected tract of superior vena cava and tracheal carina.

Traction sutures are placed in the left lateral wall of the trachea and in the median wall of the right main bronchus; the right main bronchus is transected and cross-field ventilation is performed through the right main bronchus; the trachea is transected and the specimen extracted; using tapes around the aorta for intermittent retraction of the aortic arch, anastomotic sutures are placed.

Left carinal pneumonectomy is performed through median sternotomy when a greater amount of the distal trachea is involved.

Dissection of trachea is started at the level of the innominate artery; the inferior portion of trachea is exposed in the interaorta-caval space; paratracheal lymph node dissection is then performed. The right pulmonary artery is pulled caudally and the origin of both mainstem bronchi exposed; subcarinal lymph node dissection is then performed. Dissection of right mainstem bronchus is performed.

Opening of the pericardium and the section of the ligamentum arteriosum allow control of the origin of the left pulmonary artery and, if necessary, of the superior pulmonary vein. The trachea is then encircled upward to the anticipated zone of section; the right mainstem bronchus is dissected up to the origin of the right upper lobe bronchus and encircled. Once it is absolutely certain that resection and reconstruction will be possible, the pulmonary vessels are divided.

Trachea and right mainstem bronchus are sectioned circumferentially during a short period of apnoea. Cross-field ventilation, possibly supported by jet ventilation, is performed through the right main bronchus; anastomosis is then completed.

Once the anastomosis is finished, dissection of the distal portion of the left main bronchus from oesophagus and inferior aspect of aortic arch is completed, allowing the removal of the operative specimen [30].

\section{Carinal resection with right upper lobectomy (or bilobectomy)}

This procedure is indicated when a tumour extends from the right upper lobe to the carina and the distal trachea and right pneumonectomy should be avoided. The gap between the distal trachea

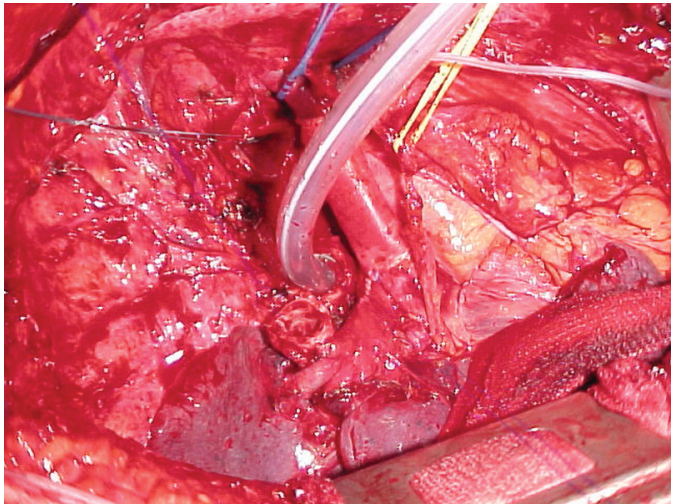

Photo 1: Intraoperative view of a carinal resection with right upper lobectomy. The trachea, the main left bronchus and the bronchus intermedius have been resected and the carina en bloc with the right upper lobe has already been removed. A cross-field intubation in the left main bronchus is maintained until the anastomosis is almost completed

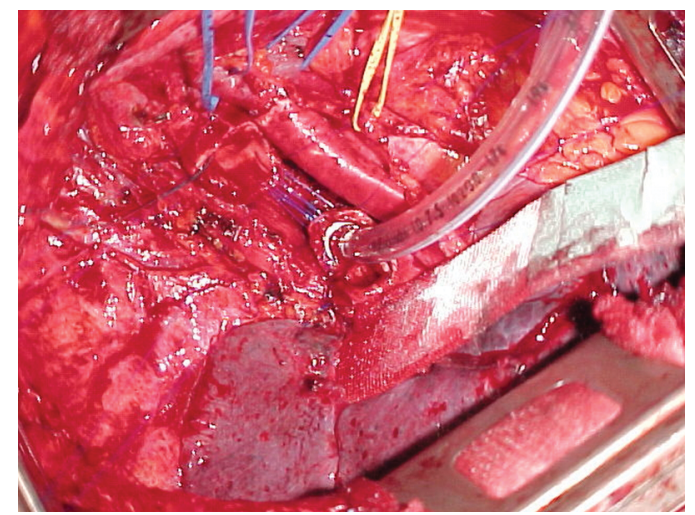

Photo 2: The anastomosis between the trachea and the main left bronchus is performed.

and the left main bronchus after excision should not exceed $4 \mathrm{~cm}$; otherwise, excessive tension on this anastomosis will result [1].

The fissure is completed first, and the vessels for the right upper lobe are legated and sectioned, before the lower trachea and carina are divided as for a right carinal pneumonectomy [28]; the bronchus intermedius is then transected below the take off of the right upper lobe bronchus (Photo 1); after that anastomosis between the trachea and the left main bronchus is completed (Photo 2), the bronchus intermedius is then anastomosed $1 \mathrm{~cm}$ below the initial anastomosis to the left main bronchus; occasionally, the bronchus intermedius can be anastomosed to the lateral wall of the trachea if the tension on this anastomosis is not excessive [28].

\section{DISCUSSION}

Carinal resection is a rare surgical procedure with a mean intervention/year rate ranging from 4.7 to 5.1 (Graph 1) [28, 31]. Thus, this procedure remains an exceptional one and should be performed in experienced centres by a skilled thoracic surgeon.

Indication to perform a carinal resection is changed over the time: to date, neoplastic disease represents the main indication (Graph 2) [28, 31]. 


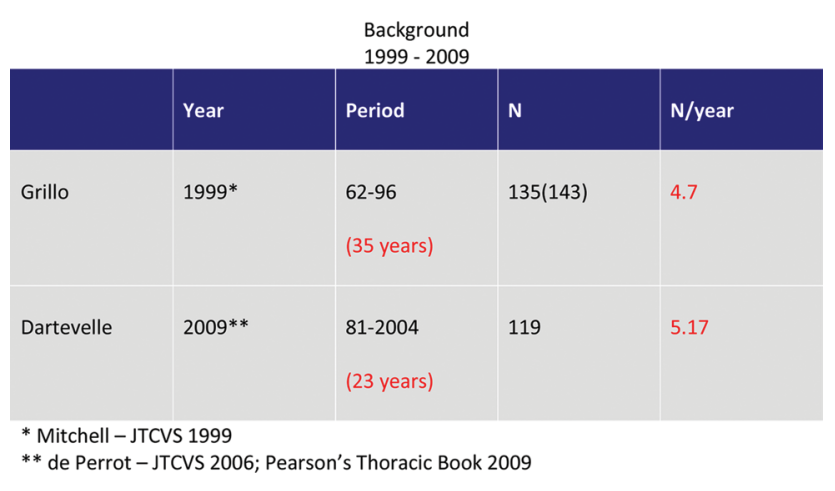

Graph 1: Table reporting the experience of two important centres about the surgery of the carina $[28,31]$.

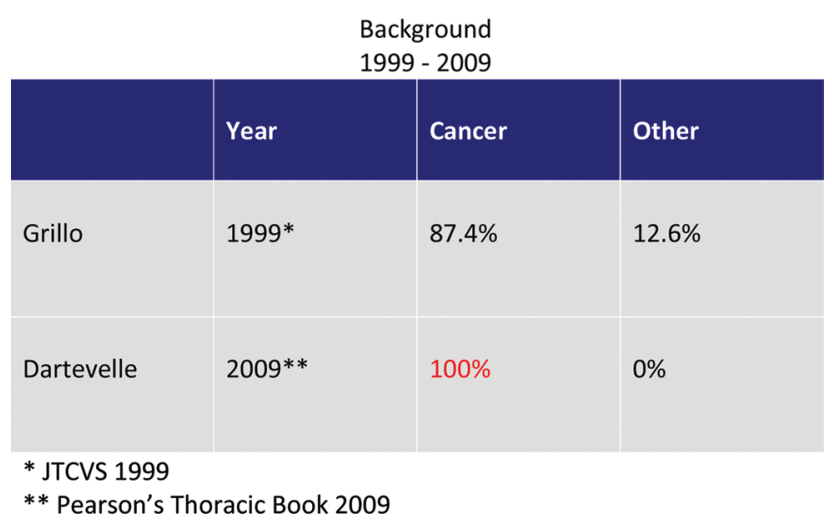

Graph 2: Table reporting how the indications for the carina's surgery changed over the time in two major centres with neoplastic disease representing the main indication.

\begin{tabular}{|c|c|c|}
\hline \multicolumn{2}{|c|}{ With lung resection } & Without lung resection \\
\hline R pn & $30.7 \%$ (1.25 pt/yr) & $36.3 \%(1.5 \mathrm{pt} / \mathrm{yr})$ \\
\hline L pn & $9 \%(0.37 \mathrm{pt} / \mathrm{yr})$ & \\
\hline Lob & $7.7 \%(0.31 \mathrm{pt} / \mathrm{yr})$ & \\
\hline $\begin{array}{l}\text { One } \\
\text { One }\end{array}$ & $\begin{array}{l}\text { heal sleeve pn ever } \\
\text { obectomy every } 3\end{array}$ & \\
\hline
\end{tabular}

Graph 3: Table reporting the Grillo's experience on the carina's surgery. Left tracheal sleeve pneumonectomy and carinal lobectomy are shown to be very exceptional procedures in this large series of carinal resection confirming that the surgery of carina is a rare surgical procedure.

Surgical procedures include carinal resection with or without parenchymal resection and as reported in the main series they are usually associated with right pneumonectomy while the left carinal pneumonectomy remains a very rare procedure with a mortality rate ranging from 2 to $16 \%$ (Graphs 3-5) [28, 31-36].

The five-year overall survival after carinal resection ranges from 26 to $44 \%$ in different series; pN0 patients present an excellent 5 -year survival rate of 50\% [31-38]. Completeness of the resection is another main factor affecting survival [32].

As already reported by Dartevelle and Macchiarini [38], induction therapy may be advocated in such patients according to the surgeon's philosophy on mediastinal node invasion, although it

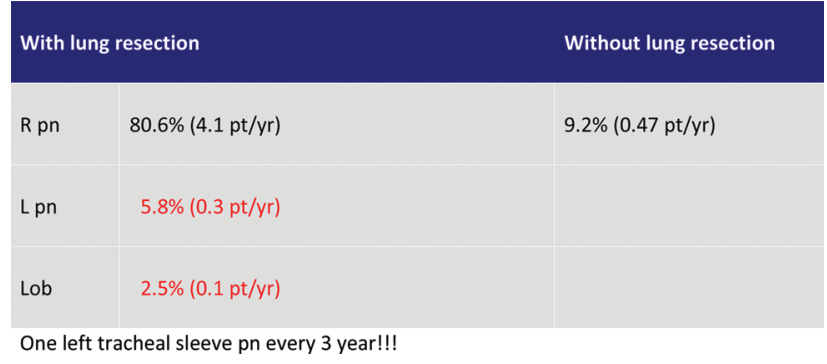

Graph 4: Table reporting the Dartevelle's experience on the carina's surgery. This series confirms the exceptionality of this surgical procedure on the carina.

\begin{tabular}{l|l|l|l|l|l|}
\multicolumn{7}{c}{ Tracheal sleeve pneumonectomy } \\
\hline & Year & N & & Pt/year & Mortality \\
\hline Mitchell & 1999 & 57 & $(44 R, 13 L)$ & 1.6 & $16 \%$ R, 30\%L \\
\hline Regnard & 2005 & 50 & $(48 R, 2 L)$ & 2.6 & $7.7 \%$ \\
\hline Roviaro & 2006 & 53 & $(52 R, 1 L)$ & 2.5 & $7.5 \%$ \\
\hline de Perrot & 2006 & 103 & $(96 R, 7 L)$ & 4.6 & $3 \% R, 0 \% L$ \\
\hline Macchiarini & 2006 & 34 & $(24 R, 10 L)$ & 5.6 & $2 \%$ \\
\hline Rea & 2008 & 49 & $(48 R, 1 L)$ & 2.1 & $6.1 \%$ \\
\hline
\end{tabular}

Graph 5: Table reporting the main series on carinal resection with or without parenchymal resection. The mortality rate in these series ranges from 2 to $16 \%$ [31-36].

increases the technical difficulties of the operation and perioperative complication rate. Ten years later, Macchiarini and the Hannover Interdisciplinary Intrathoracic Tumour task force group advocated surgical treatment in such patients in case of PETnegative mediastinal nodes and chemo-radiotherapy in $\mathrm{pN} 2+$ patients at mediastinoscopy, possibly followed by surgery only in case of no progressive disease at re-evaluation [35].

In case of T4 non-small cell lung cancer (NSCLC) with carinal involvement, induction chemotherapy may be advocated to reduce the rate of explorative thoracotomy, the rate of positive margins after resection as well as systemic recurrences leading to an unsatisfactory survival outcome.

Theoretical advantages of induction therapy in these patients are the early control of systemic micrometastases, the downstaging of unresectable disease, the increasing of resectability and complete resection as well as the rate of parenchyma sparing procedure, thus reducing the amount of extended resections.

Better compliance than adjuvant therapy as well as drug delivering to loco-regional disease through an intact vascular bed should also be considered.

On the other hand, theoretical disadvantages like delay in local control, increase in surgical difficulties and increase in morbidity and mortality rate should be taken into consideration.

We personally consider absolute indications to induction chemotherapy in the case of carinal involvement, the presence of superior vena cava involvement as well as cN2 disease.

In our personal series of 37 carina resections, we performed 26 right tracheal sleeve pneumonectomies, 3 right upper lobectomies with carina resection, 7 carina resections with neocarina, 1 carinal resection according to reverse Barclay technique 
(Eschapasse). The overall 5-year survival rate is $27 \%$ with a splitted 5 -year survival rate of $32 \%$ in down-staged patients and of $12.5 \%$ in not down-staged patients.

\section{CONCLUSIONS}

Carinal resection for cancer is feasible and presents a low-rate of postoperative mortality when performed in specialized centres. Very good survival results can be obtained, especially with high selected patients ( $\mathrm{pN} 0$ or $\mathrm{pN} 1$ ).

Some open questions still remain debatable: left tracheal sleeve pneumonectomy and carinal lobectomy may be overcome by combined treatments with new drugs and radiotherapy; induction treatment may be considered the gold standard base line treatment in carinal disease or as mandatory approach for cN2 disease.

\section{Conflict of interest: none declared.}

\section{REFERENCES}

[1] Grillo HC. Surgery of the Trachea and Bronchi, 1st edn. Hamilton, London: BC Decker Inc., 2004.

[2] Grindlay JH, Clagett OT, Moersch HJ. Experimental surgery of the thoracic trachea and its bifurcation; preliminary report. Proc Staff Meet Mayo Clin 1949;24:555

[3] Juvenelle AA, Citret $C$. Transplantation de la bronche souche et résection de la bifurcation trachéale. Une étude expérimentale sur le chien. J Chir 1951;67:666-88

[4] Kiriluk LB, Merendino KA. An experimental evaluation in the dog of bronchial transplantation, bronchial, tracheal and tracheobronchial resection with reconstruction. Ann Surg 1953;137:490-503.

[5] Ehrlich RW, Meyer RP, Taylor C, Hass GM, Miller EM. Reconstruction of the tracheobronchial tree with resection of the lung, carina and lower trachea; an experimental study. Surg Gynecol Obstet 1952;94:570-6.

[6] Abbott OA. Experiences with the surgical resection of the human carina tracheal wall and contralateral bronchial wall in cases of right total pneumonectomy. J Thorac Surg 1950;19:906-22.

[7] Mathey J. Tumeur bénigne de l'éperon Trachéal. Résection et Réfection du carrefour trachéo-bronchique. La Sem des Hopitaux Paris 1951;27:2699-703.

[8] Crafoord C, Bjork VO, Hilty H. Bronchial resection and broncho-tracheal anastomosis in tuberculous bronchial stenosis; report of a case. Thoraxchirurgie 1954;2:21-7.

[9] Bjork VO. Left sided bronchial anastomosis. J Thorac Surg 1955;30:492-8.

[10] Barclay RS, McSwan N, Welsh TM. Tracheal reconstruction without the use of grafts. Thorax 1957;12:177-80.

[11] Grillo HC, Bendixen HH, Gephart T. Resection of the carina and lower trachea. Ann Surg 1963;158:889-93.

[12] Nissen R. Extracorporeal circulation for prolonged (30 min) respiratory interruption in surgery of tracheal tumors in the area of the bifurcation. Schweiz Med Wochenschr 1961;91:957-64.

[13] Woods FM, Neptune WB, Palatichi A. Resection of the carina and mainstem bronchi with the use of extracorporeal circulation. N Engl J Med 1961;264:492-4

[14] Eschapasse H, Vahdat F, Gaillard J, Besso JC. Reflections on resection of the lower trachea and bronchial bifurcation. Ann Chir Thorac Cardiovasc 1967;6:63-70.
[15] Grillo HC. Carinal reconstruction. Ann Thorac Surg 1982;34:356-73.

[16] Klepetko W, Marta GM, Wisser W, Melis E, Kocher A, Seebacher G et al. Heterotopic tracheal transplantation with omentum wrapping in the abdominal position preserves functional and structural integrity of a human tracheal allograft. J Thorac Cardiovasc Surg 2004;127:862-7.

[17] Macchiarini P, Walles T, Biancosino $C$, Mertsching $H$. First human transplantation of a bioengineered airway tissue. J Thorac Cardiovasc Surg 2004;128:638-41.

[18] Macchiarini P, Jungebluth P, Go T, Asnaghi MA, Rees LE, Cogan TA et al. Clinical transplantation of a tissue-engineered airway. Lancet 2008;372:2023-30.

[19] Smith RA, Nigam BK. Resection of proximal left main bronchus cancer. Thorax 1979;34:616-20.

[20] Maeda M, Nakamoto K, Tsubota N, Okada T, Katsura H. Operative approaches for left-sided carinoplasty. Ann Thorac Surg 1993;56:441-6.

[21] Goeltz FA. Successful anterior thoraco-bronchotomy for a foreign body impacted in the bronchus. Ann Surg 1907;43:369-72.

[22] Padhi RK, Lynn RB. The management of bronchopleural fistulas. J Thorac Cardiovasc Surg 1960;39:385-93.

[23] Abruzzini P. Trattamento chirurgico delle fistole del bronco principale consecutive a pneumonectomia per tubercolosi. Chir Toracica 1961;14:165-71.

[24] Perelman MI. Surgery of the Trachea. Moscow: Mir, 1976.

[25] Reitz BA, Wallwork JL, Hunt SA, Pennock JL, Billingham ME, Oyer PE et al. Heart-lung transplantation: successful therapy for patients with pulmonary vascular disease. N Engl J Med 1982;306:557-64.

[26] Rouvière H. Anatomy of the Human Lymphatic System. In: Tobias MJ (trans). Ann Arbor, MI: Edwards Bros., 1938.

[27] Riquet M, Le Pimpec Barthes F, Souilamas R, Hidden G. Thoracic duct tributaries from intrathoracic organs. Ann Thorac Surg 2002;73:892-9.

[28] de Perrot M, Fadel E, Dartevelle P. Carinal resection, In: Patterson GA, Cooper JD, Deslauriers J, Lerut AEM, Luketich JD, Rice TW (eds). Pearsons's Thoracic and Esophageal Surgery, 3rd edn. Churchill, PA: Livingstone Elsevier, 2008, 383-92.

[29] Salzer GM, Müller LC, Kroesen G. Resection of tracheal bifurcation through a left thoracotomy. Eur J Cardiothorac Surg 1987;1:125-8.

[30] Alifano M, Regnard JF. Sleeve pneumonectomy. MMCTS 2007 2007:mmcts.2006.002113; doi:10.1510/mmcts.2006.002113.

[31] Mitchell JD, Mathisen DJ, Wright CD, Wain JC, Donahue DM, Moncure AC et al. Clinical experience with carinal resection. J Thorac Cardiovasc Surg 1999;117:39-52.

[32] Regnard JF, Perrotin C, Giovannetti R, Schussler O, Petino A, Spaggiari L et al. Resection for tumors with carinal involvement: technical aspects, results, and prognostic factors. Ann Thorac Surg 2005;80:1841-6.

[33] Roviaro G, Vergani C, Maciocco M, Varoli F, Francese M, Despini L. Tracheal sleeve pneumonectomy: long-term outcome. Lung Cancer 2006;52:105-10.

[34] de Perrot M, Fadel E, Mercier O, Mussot S, Chapelier A, Dartevelle P. Long-term results after carinal resection for carcinoma: does the benefit warrant the risk? J Thorac Cardiovasc Surg 2006;131:81-9.

[35] Macchiarini P, Altmayer M, Go T, Walles T, Schulze K, Wildfang I et al. Hannover Interdisciplinary Intrathoracic Tumor Task Force Group. Technical innovations of carinal resection for non-small cell lung cancer. Ann Thorac Surg 2006;82:1989-97; discussion 1997.

[36] Rea F, Marulli G, Schiavon M, Zuin A, Hamad AM, Feltracco P et al. Tracheal sleeve pneumonectomy for non small cell lung cancer (NSCLC): short and long-term results in a single institution. Lung Cancer 2008;61:202-8.

[37] Mitchell JD, Mathisen DJ, Wright CD, Wain JC, Donahue DM, Allan JS et al. Resection for bronchogenic carcinoma involving the carina: long-tersm results and effect of nodal status on outcome. J Thorac Cardiovasc Surg 2001;121:465-71.

[38] Dartevelle P, Macchiarini P. Techniques of pneumonectomy. Sleeve pneumonectomy. Chest Surg Clin N Am 1999;9:407-17. 